\title{
Comparison of Glomerular Filtration Rate Estimated by Three Methods in a Pakistani Community Cohort
}

\author{
Ashar Alam, Sundus Iftikhar, and Naila Baig-Ansari
}

\section{ABSTRACT}

\begin{abstract}
Background: Estimated glomerular filtration rate (eGFR) has always been considered a better and more accurate method to assess kidney function as compared to serum creatinine. Various equations such as the CockroftGault equation corrected for surface area (CG-CRTD), the four-variable Modification of Diet in Renal Disease equation (MDRD) and 2009 Chronic Kidney Disease Epidemiology Collaboration equation (CKD-EPI) have been derived to estimate GFR. In the South Asian region, the CKD-EPI equation has not been validated or compared with other equations. This study compares eGFR in a Pakistani community cohort calculated by all three equations.
\end{abstract}

Results: Serum creatinine was measured using Kinetic Colorimetric Assay in alkaline medium for $\mathbf{4 6 1}$ individuals age $\mathbf{1 5}$ and above. Less than half of the participants (47\% by CG-CRTD, $40 \%$ by MDRD, $52 \%$ by CKD-EPI) had eGFR $\geq 90 \mathrm{ml} / \mathrm{min}$ per $1.73 \mathrm{~m}^{2}$ and for participants with eGFR $\geq 120$ $\mathrm{ml} / \mathrm{min}$ per $1.73 \mathrm{~m} 2$, the percentages were even lower $(15 \%, 12 \%$, and $22 \%$ respectively). All the equations were linearly associated with each other, but the error estimation depicted that majority of the individual differences were $\geq 5$ and $\leq-5$, and very few were within \pm 1 indicating less degree of agreement between the formulas. Age was significantly but negatively correlated with all the three formulas in their classification of patients as per eGFR Conclusion: Our study found that the values of estimated GFR are on lower side for Pakistanis as compared to the western population. The equations available for estimation of GFR even though associated linearly with each other have significant individual differences. Thirdly, using eGFR to classify CKD should have a better consideration of physiological agerelated decline in GFR. All of these findings necessitate having an adequately funded randomized study measuring true GFR of this population and at the same time validating eGFR equations to find the normal GFR ranges of South Asians.

Keywords: calculated GFR, chronic kidney disease, estimated GFR, Pakistan.
Published Online: June 26, 2021

ISSN: $2736-5476$

DOI: $10.24018 /$ ejclinicmed.2021.2.3.60

Ashar Alam*

Department of Nephrology, The Indus Hospital, Korangi Crossing, Karachi, Pakistan.

(e-mail: ashar.alam ${ }^{\circledR}$ tih.org.pk) Sundus Iftikhar

Maternal and Child Health Program, IRD Pakistan, Karachi, Pakistan.

(e-mail: sundus.iftikhar@ird.global) Naila Baig-Ansari

Indus Hospital Research Center (IHRC), The Indus Hospital, Korangi Crossing, Karachi, Pakistan

(e-mail: naila.baigansari@tih.org.pk)

*Corresponding Author

\section{INTRODUCTION}

Non-communicable diseases (NCDs) present a significant global health challenge in the current century and have replaced communicable diseases as the most common causes of morbidity and premature mortality worldwide. The chronic kidney disease (CKD) has emerged now as a separate NCD and requires classification based on glomerular filtration rate (GFR). The measurement of true GFR is cumbersome and requires iothalamate or some other suitable substance to be injected into the human body. Therefore, various recognized organizations recommend that clinical laboratories report estimated GFR (eGFR) that provide more accurate assessment of the level of kidney function than serum creatinine alone [1]-[3]. Multiple equations have been derived to estimate GFR including the earliest Cockroft-Gault equation (CG-CRTD) [4], the later Modification of Diet in Renal Disease study equation (MDRD) [5] and the 2009 Chronic Kidney Disease Epidemiology Collaboration equation (CKD-EPI) [6]. Validation studies have also been performed on these equations mostly in western parts of the world [3], [7]-[11]. In South Asia that makes around 23.5\% of the whole world population according to latest World Bank data available online, a few studies [12]-[15] had compared Cockroft-Gault and MDRD equations but CKD-EPI equation that is relatively newer has never been validated or compared with other equations in this region.

We analyzed the results of the Indus Hospital Community Cohort (IHCC) that was established in last decade with the objective of having a unique 'Framingham-like' Pakistani cohort to investigate the prevalence and risk factors for hypertension, obesity, diabetes, coronary artery disease, kidney disease and hepatitis $\mathrm{B}$ and $\mathrm{C}$ infection in a multi- 
ethnic, middle to low income population. The kidney function was assessed by GFR that was estimated using multiple equations available to-date. The paper highlights the comparison of estimated GFR by three methods in a South Asian adult cohort population.

\section{METHODS}

Six hundred and sixty-seven households were enrolled between March 2010 and August 2011 including 461 adults in the catchment area of The Indus Hospital's community cohort in Karachi, Pakistan. Detailed methodology of the cohort [16] as well as the baseline findings of prevalence and risk factors of kidney disease in urban Karachi [17] have been published elsewhere.

The baseline survey included questionnaires, anthropometric measurements, physical examination as well as ultrasound and laboratory assessment. Serum creatinine was measured using Kinetic Colorimetric Assay in alkaline medium on Hitachi 902 (Japan). The glomerular filtration rate (GFR) was estimated by three methods:

The earliest Cockcroft-Gault Equation as follows:

$$
\begin{aligned}
& \mathrm{GFR}(\mathrm{CG})=(140- \text { age }) \times \mathrm{Wt}[\mathrm{kg}] \\
& \times \frac{(0.85 \text { if female })}{\left(72 \times \text { serum } \mathrm{Cr}\left[\frac{\mathrm{mg}}{\mathrm{dl}}\right]\right)}
\end{aligned}
$$

It was then corrected for Body Surface Area (BSA) that was calculated according to the Dubois and Dubois formula:

$$
\begin{gathered}
\text { BSA }\left[\mathrm{m}^{2}\right]=0.20247 \times \text { height }[\mathrm{m}] 0.725 \\
\times \text { weight }[\mathrm{kg}] 0.425 \\
\text { CG }- \text { CRTD }=\text { GFR }(\mathrm{CG}) \times \frac{1.73}{B S A}
\end{gathered}
$$

The newer 4-variable Modification of Diet in Renal Disease (MDRD) study equation as follows:

$$
\begin{aligned}
\operatorname{MDRD}=186 \times & \left(\text { serum } \mathrm{Cr}\left[\frac{m g}{\mathrm{dl}}\right]\right)-1.154 \times \text { age } \\
& -0.203 \times(0.742 \text { if female })
\end{aligned}
$$

The latest CKD-EPI equation as follows:

CKD-EPI $=141 \times \min ($ serum $\mathrm{Cr} / \kappa, 1) \alpha \times \max ($ serum $\mathrm{Cr} / \kappa, 1)-1.209 \times 0.993 \mathrm{Age} \times(1.018$ if female $) \times(1.159$ if black):

$$
\begin{aligned}
\mathrm{CKD}-\mathrm{EPI} & =141 \times \min \left(\operatorname{serum} \frac{\mathrm{Cr}}{\kappa}, 1\right) \alpha \\
& \times \max \left(\operatorname{serum} \frac{\mathrm{Cr}}{\kappa}, 1\right) \\
& -1.209 \times 0.993 \text { Age } \\
& \times(1.018 \text { if female }) \times(1.159 \text { if black })
\end{aligned}
$$

where $\kappa$ is 0.9 for males and 0.7 for females, $\alpha$ is -0.411 for males and -0.329 for females, min indicates the minimum of serum $\mathrm{Cr} / \kappa$ or 1 and max indicates the maximum of serum $\mathrm{Cr} / \kappa$ or 1 .
Body mass index (BMI) was derived by dividing weight (in $\mathrm{kg}$ ) by height squared (in $\mathrm{m}^{2}$ ). Abnormal BMI level was set as $\geq 25 \mathrm{~kg} / \mathrm{m}^{2}$ both in males and females.

Statistical analyses were carried out using SPSS version 21 and R software. Shapiro Wilk's test and skewness values were used to check normality of quantitative variables age, weight, height, BMI, GFR estimations, BSA and creatinine. Mean (SD) was computed for all the quantitative variables. Frequency and percentage were computed for gender. Repeated measures ANOVA was applied to check significant differences among the three GFR estimation methods. Gwet's AC1 statistics for GFR classification into CKD stages were used to measure agreement between the three methods. To assess differences in individual results calculation of the error, Bland- Altman 95\% limits of agreement tests and Passing Bablok regression analysis were used. Error was defined as the difference between the GFR values calculated by three equations. Better agreement between the methods was defined as the smaller Bland and Altman 95\% limits of agreement, points closer to zero across the entire range of mean scores in Bland-Altman plots, at least $50 \%$ of the differences (errors) lying within \pm 1 , no systematic differences (95\% CI of intercept of Passing Bablok regression contains zero), no proportional differences (95\% CI of slope of Passing Bablok regression contains 1) and no random differences (smaller \pm 1.96 CI of Residual standard deviations). Pearson correlation was used to check association of age and BMI with CKD stages. P-value $<0.05$ was considered significant.

This study was approved by the Institutional Review Board (IRB) of Interactive Research and Development (IRD). Written informed consent was obtained from all participants.

This study was supported by separate grants from Interactive Research \& Development (IRD), the Association of Pakistani-Descent Cardiologists of North America (APCNA) and the International Society of Nephrology Commission for the Global Advancement of Nephrology (ISN-COMGAN).

All the authors declared no competing interests. The study was partly supported by a grant of International Society of Nephrology (ISN).

\section{RESULTS}

A total of 461 adults (15 years of age and older) took part in the IHCC baseline survey. However, 46 of them could not submit their blood sample for serum creatinine and 5 more did not submit height and weight. The socio-economic statistics of those excluded were similar to those who were included in the analysis. GFR of the remaining 410 adults was calculated by all three methods mentioned earlier.

Out of these 410 participants, 147 (35.9\%) were males and $263(64.1 \%)$ were females. Mean \pm SD age of the participants was $34.5 \pm 15$ (males: $35.8 \pm 16.5$ years and females: $33.7 \pm 14.1$ years). The baseline characteristics of the participants are shown in Table I.

\section{A. Agreement between the Methods}

Significant differences were found in mean vales among the three GFR estimation formulas (Table I).

The patients were further grouped into three categories as 
per estimated GFR; 1) eGFR $<60 \mathrm{ml} / \mathrm{min}, 2$ ) eGFR $\geq 60$ but $<$ $90 \mathrm{ml} / \mathrm{min}$, 3) eGFR $\geq 90 \mathrm{ml} / \mathrm{min}$. Agreement between the formulas in their classification of patients as per eGFR was evaluated b'y Gwet's AC1 statistics. According to this there was substantial agreement between CG-CRTD and CKD EPI (0.68) and MDRD and CG-CRTD (0.61) whereas strong agreement was observed between MDRD and CKD-EPI (0.83) (Table not shown).

Individual differences among the formulae were assess by calculating error (differences between the values), Bland Altman 95\% limits of agreement and Passing Bablok regression. Table 2 shows the frequency of residual computed from the data using the three formulas. It showed that the difference between the individual values of the formulas was huge. Majority of the differences were $\geq 5$ and $\leq-5$; very few were within \pm 1 indicating less degree of agreement between the formulas (Table II).

Also, it is evident from Bland Altman plots (Figure 1) that the data points are widely dispersed across the zero difference line, indicating a lot of variation between measurements. A number of outliers are also evident in figures $1 \mathrm{a}-1 \mathrm{c}$. The $95 \%$ limits of agreement are also very much wide that are not clinically acceptable showing greater variations in the differences and less agreement between the measurements. Hence, indicating that none of the method can be used in place of other.

Passing Bablok regression analysis shows that none of the three methods are comparable as \pm 1.96 RSD interval is wide enough, also systematic and proportional differences exists between CKD-EPI and MDRD, where proportional differences exists between MDRD and CG-CRTD (Table III).

\section{B. Association between Classifications as per eGFR and Patients' Characteristics}

Age was found to be significantly but negatively correlated with all the three formulas in their classification of patients as per eGFR (MDRD:-0.565, CG-CRTD: -0.609, CKD-EPI: 0.661; p-value<0.0001), however BMI was found to be significantly positively correlated with CG-CRTD (0.244; Pvalue $=0.244)$ and negatively correlated with the other two methods (MDRD: -0.233 , CKD-EPI $=-0.238$; $\quad \mathrm{P}$ value $<0.0001)$.

TABLE I: CHARACTERISTICS OF STUDY POPULATION

\begin{tabular}{lc}
\hline Gender; $\mathrm{n}(\%)$ & $147(35.9)$ \\
$\quad$ Male & $263(64.1)$ \\
$\quad$ Female & Mean \pm SD \\
& $34.5 \pm 15$ \\
Age (years) & $54.9 \pm 12.8$ \\
Height $(\mathrm{cm})$ & $156.9 \pm 11$ \\
Weight $(\mathrm{Kg})$ & $22.3 \pm 5.1$ \\
BMI $\left(\mathrm{kg} / \mathrm{m}^{2}\right)$ & $1.5 \pm 0.2$ \\
BSA $\left(\mathrm{m}^{2}\right)$ & $0.94 \pm 0.23$ \\
Creatinine $(\mathrm{mg} / \mathrm{dl})$ & \\
GFR & $90.2 \pm 23.1$ \\
CG $\left(\mathrm{ml} / \mathrm{min}\right.$ per $\left.1.73 \mathrm{~m}^{2}\right)$ & $87.4 \pm 23$ \\
MDRD $\left(\mathrm{ml} / \mathrm{min}\right.$ per $\left.1.73 \mathrm{~m}^{2}\right)$ & $91.9 \pm 22.2$ \\
CKD-EPI $\left(\mathrm{ml} / \mathrm{min}\right.$ per $\left.1.73 \mathrm{~m}^{2}\right)$ & \\
\hline
\end{tabular}

a. Statistically significant difference in mean values of GFR among the three equations.
TABLE II: COMPARISON OF THE PERFORMANCE OF THREE GFR

\begin{tabular}{ccccccc}
\multicolumn{9}{c}{ Error } & \multicolumn{2}{c}{ CKD-EPI, } & \multicolumn{2}{c}{$\begin{array}{c}\text { CKD-EPI, CG- } \\
\text { CRTD }\end{array}$} & \multicolumn{2}{c}{$\begin{array}{c}\text { CG-CRTD, } \\
\text { MDRD }\end{array}$} \\
\cline { 2 - 7 } & $\mathrm{n}$ & $\%$ & $\mathrm{n}$ & $\%$ & $\mathrm{n}$ & $\%$ \\
\hline$<=-5$ & 16 & 3.9 & 110 & 26.8 & 114 & 27.8 \\
-4.99 to -4 & 5 & 1.2 & 9 & 2.2 & 12 & 2.9 \\
-3.99 to -3 & 7 & 1.7 & 6 & 1.5 & 15 & 3.7 \\
-2.99 to -2 & 10 & 2.4 & 11 & 2.7 & 10 & 2.4 \\
-1.99 to -1 & 11 & 2.7 & 11 & 2.7 & 12 & 2.9 \\
-0.99 to 0 & 8 & 2 & 20 & 4.9 & 15 & 3.7 \\
0.01 to 0.99 & 9 & 2.2 & 13 & 3.2 & 12 & 2.9 \\
1 to 1.99 & 20 & 4.9 & 12 & 2.9 & 11 & 2.7 \\
2 to 2.99 & 21 & 5.1 & 15 & 3.7 & 12 & 2.9 \\
3 to 3.99 & 22 & 5.4 & 20 & 4.9 & 11 & 2.7 \\
4 to 4.99 & 35 & 8.5 & 12 & 2.9 & 8 & 2 \\
$>=5$ & 246 & 60 & 171 & 41.7 & 178 & 43.4 \\
Within $\pm \mathbf{1}$ & 17 & 4.1 & 33 & 8.0 & 27 & 6.6 \\
Within $\pm \mathbf{2}$ & 48 & 11.7 & 56 & 13.7 & 50 & 12.2 \\
\hline
\end{tabular}

TABLE III: PASSING BABLOK REGRESSION ANALYSIS CKD-EPI (x) vs CKD-EPI (x) MDRD (x) vs CG-CRTD (y) vs MDRD (y) CG-CRTD (y)

* Intercept 95\% CI does not contain zero; indicating systematic differences. ** Slope $95 \%$ CI does not contain 1; indicating proportional differences. $\ddagger \pm 1.96 \mathrm{RSD}$ interval are wide enough; indicating the two methods are not comparable.

Bland-Altman plots- differences between the two methods are plotted against the mean values of the two methods. Limits of Agreement (Upper Confidence Limit and Lower Confidence Limit) calculated as mean difference \pm 2 SD of the difference.

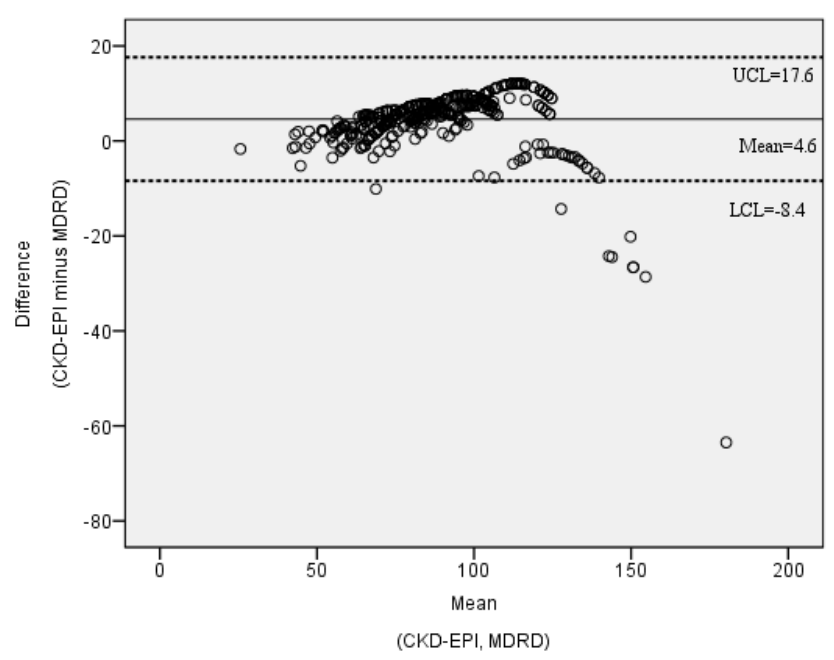

Fig. 1a: Bland-Altman plot: Differences between CKD-EPI and MDRD. 


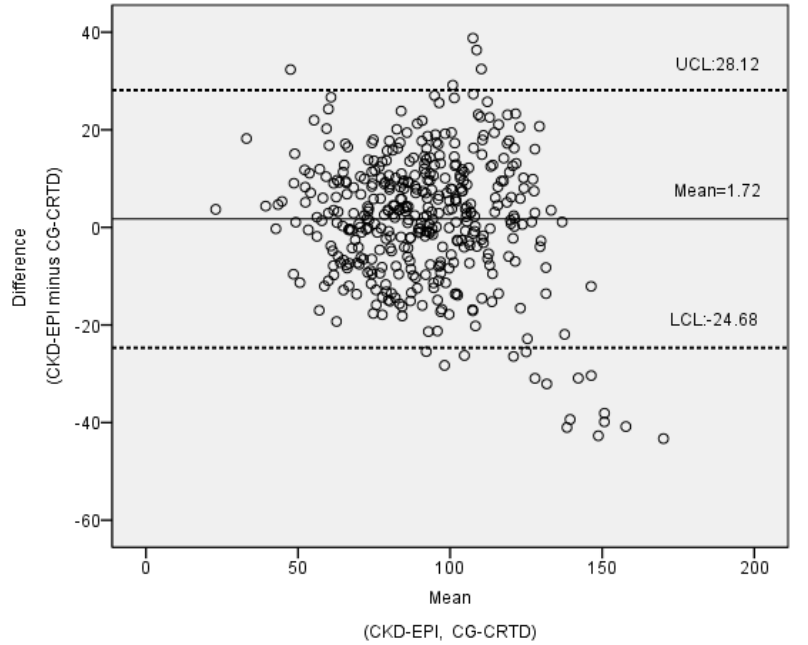

Fig. 1b: Bland-Altman plot: Differences between CKD-EPI and CG-CRTD.

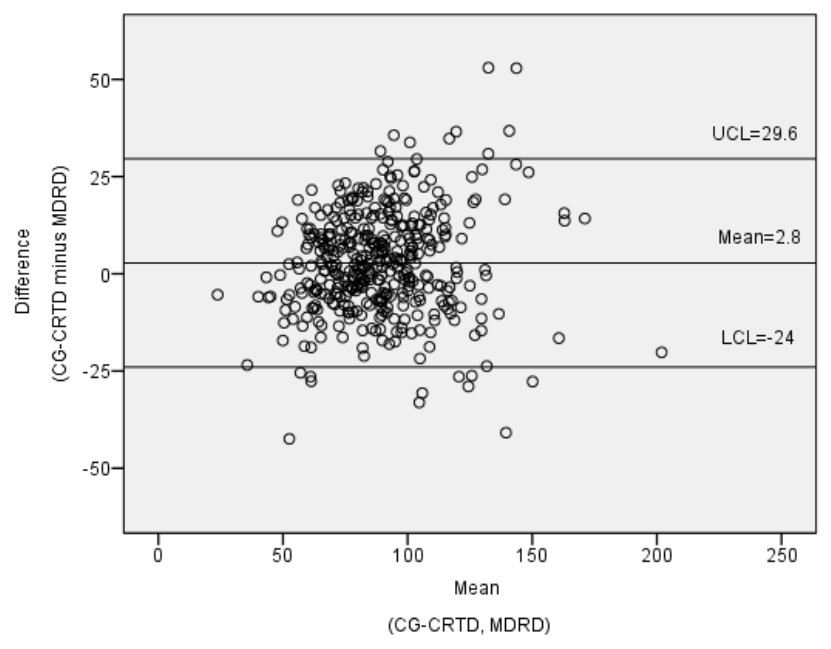

Fig. 1c: Bland-Altman plot: Differences between CG-CRTD and MDRD.

\section{DISCUSSION}

Estimated GFR (eGFR) has always been considered a better and more accurate method to assess kidney function as compared to serum creatinine. National and international organizations recommend that clinical laboratories report eGFR and that clinicians use this to evaluate kidney function for all patients [1]-[3]. Various equations have been derived to calculate eGFR including Cockroft-Gault [4],modified MDRD [5] and the CKD-EPI 2009 [6] equation. Validation studies have also been performed on these equations in various parts of the world [3], [7]-[11]. In South Asian region that comprises around one-fourth of the world population, at least one study each from India[14] and Bangladesh [15] was published comparing GFR estimated by Cockroft-Gault and modified MDRD study equations with GFR measured with 99mTc-diethylenetriamine penta-acetic acid (DTPA) renogram. One study from Pakistan [13] had tested these two equations against 24-hour urinary creatinine clearance, but not the measured GFR. However, CKD-EPI 2009 equation has never been validated or compared with other equations in this region.

This is the first published study from Pakistan that has compared estimated GFR by all three methods in a community cohort. Average $( \pm \mathrm{SD})$ age, BMI, serum creatinine and BSA of our study participants was 34.5 years $( \pm 15), 22.3 \mathrm{~kg} / \mathrm{m}^{2}( \pm 5.1), 0.94 \mathrm{mg} / \mathrm{dl}( \pm 0.23)$ and $1.5 \mathrm{~m}^{2}$ $( \pm 0.2)$, respectively. Mean $( \pm \mathrm{SD})$ eGFR was $90.2( \pm 23.1)$, $87.4( \pm 23$.) and $92( \pm 22)$, by corrected Cockroft-Gault, modified MDRD and CKD-EPI methods respectively. Only one study was published from Pakistan[18] that had measured GFR using urinary clearance of Inulin on 530 participants and found mean GFR $94.1 \pm 28.6 \mathrm{ml} / \mathrm{min}$ per $1.73 \mathrm{~m}^{2}$. However, the said study was restricted to adults aged 40 years or older, the mean GFR had a wide standard deviation/dispersion. The studies from India [14] and Bangladesh [15] measuring GFR with $99 \mathrm{mTc}$-diethylenetriamine penta-acetic acid (DTPA) renogram had found it to be $95.5 \pm 11.6$ and $85.4 \pm 14.8 \mathrm{ml} / \mathrm{min}$ per $1.73 \mathrm{~m}^{2}$, respectively. All of these studies still suggest that the normal GFR seems to be lower in the South Asian population compared to the western standards. But whether the estimated GRF also reflects same trend or not, is unknown. Our study shows the results of eGFR by all three methods and variation among themselves in same population.

Our findings showed that less than half of the participants ( $47 \%$ by CG-CRTD, $40 \%$ by MDRD, $52 \%$ by CKD-EPI) had eGFR $\geq 90 \mathrm{ml} / \mathrm{min}$ per $1.73 \mathrm{~m}^{2}$ and even lower for participants having eGFR $\geq 120 \mathrm{ml} / \mathrm{min}$ per $1.73 \mathrm{~m}^{2}$, the normal value cutoff for GFR in western populations. A significant percentage of participants had eGFR 60 to $89 \mathrm{ml} / \mathrm{min}$ per $1.73 \mathrm{~m}^{2}(43 \%$ by CG-CRTD, $51 \%$ by MDRD, $41 \%$ by CKD-EPI), while few had eGFR $<60 \mathrm{ml} / \mathrm{min}$ per $1.73 \mathrm{~m}^{2}$ (9.5\% by CG-CRTD, $8 \%$ by MDRD, $7 \%$ by CKD-EPI). Having significant number of subjects in eGFR 60 to $89 \mathrm{ml} / \mathrm{min}$ per $1.73 \mathrm{~m}^{2}$ group raises concerns that 'normal' value of GFR in our population may be lower than those calculated in western countries. This can be due to different body physique of South Asian population. Similar findings of having an eGFR $<90 \mathrm{ml} / \mathrm{min}$ per $1.73 \mathrm{~m}^{2}$ by $\mathrm{CG}$ and MDRD formula in more than $50 \%$ of the study subjects were found in two Indian studies [12], [19].

In our study, the participants were distributed into three groups as per eGFR calculated by CKD-EPI method and their eGFR values by other two methods were compared in each group. Substantial agreement of CKD-EPI was observed with CG-CRTD and MDRD, whereas there was strong agreement between CG-CRTG and MDRD. The correlation between these two equations had been studied outside South Asia in detail [20], [21] and a very good correlation between eGFR estimated by CKD-EPI and MDRD at values less than $60 \mathrm{ml} / \mathrm{min}$ per $1.73 \mathrm{~m}^{2}$ has been demonstrated, but not at higher values. The MDRD equation underestimated GFR at levels greater than $60 \mathrm{ml} / \mathrm{min}$ per $1.73 \mathrm{~m}^{2}$, therefore using CKD-EPI equation resulted in a lower estimated prevalence of CKD and improved risk prediction, compared to the MDRD equation [22]-[24].Due to very same reason, many laboratories do not report estimated GFR (eGFR) at values more than $60 \mathrm{ml} / \mathrm{min}$ per $1.73 \mathrm{~m}^{2}$. Unfortunately, no such correlation has been studied so far in the South Asian population.

Further analysis in our study revealed that the difference between the individual results of equations was huge. This was a very interesting finding that was proved by three statistical tests in our study. Error Estimation depicted that majority of the individual differences were $\geq 5$ and $\leq-5$, and very few were within \pm 1 indicating less degree of agreement between the formulas. Secondly, Bland Altman Plots showed 
that the data points were widely dispersed across the zero difference line, indicating a lot of variation between measurements, however, lesser disagreement was found between MDRD and CKD-EPI methods. Lastly, Passing Bablok Regression Analysis showed systematic, proportional and random differences between various methods and concluded that none of any two methods were comparable.

Our study found that age was significantly but negatively correlated with all the three formulas in their classification of patients as per eGFR. Women were found to have a slightly higher eGFR. Poggio et al [25] have elaborated this impact of age and gender on GFR while evaluating living kidney donors at the Cleveland Clinic from 1972 to 2005. It is well known that this decline of GFR with age appears to be a part of the normal physiologic process of cellular and organ senescence and does not reflect underlying chronic parenchymal injury on renal biopsy [26], [27].But the problem is that KDIGO considers fixed cut-offs while classifying CKD and links prognosis to CKD class. It has been pointed out earlier [28] that these classes and prognosis may be more valuable and meaningful if the parameter 'age' (senescence) is better taken into consideration. Otherwise, many individuals over age 65 may be labeled as having CKD even though their GFR is within the normal range for their age and gender. .For this reason certain researchers have even shown serious doubts on the currently known prevalence of CKD and have also shown concern about the routine reporting of eGFR every time a serum creatinine is measured [27]. Our study certainly shows that eGFR decreases with age but how much of it is pathological (if any) is not known, nor can be figured out through these equations.

We also found that BMI was significantly positively correlated with CG-CRTD and negatively correlated with the other two methods. There has been conflicting data in previous studies with respect to this association. A recent study done on 491 subjects from South India [12] had shown the same trend as that of us. It had also been described in other studies that higher BMI was consistently associated with lower MDRD [29] and CKD-EPI [30], while a few others had depicted a non-linear relationship between eGFR decline per year and BMI [31], [32]. We believe that the relation between BMI and eGFR is not well known and the variability in predictions of kidney function by eGFR depend on age, BMI and other factors resulting in conflicting data among the cohorts in past studies.

Although the available literature [33], [34] and the Kidney Disease: Improving Global Outcomes (KDIGO) 2012 Clinical Practice Guideline for the Evaluation and Management of Chronic Kidney Disease [35] clearly recommends the CKD-EPI creatinine equation as the most accurate method for estimating GFR for diverse populations and supports that CKD-EPI equation should replace MDRD study equation for general use. Compared with the MDRD Study equation, the CKD-EPI equation permits more accurate GFR estimation, fewer false-positive diagnoses of CKD and lower prevalence estimates for CKD. On the other hand, it has also been found in detailed reviews [36], [37] that no single equation can claim to be valid for every specific group of individuals, and the direct measurement of 'true' GFR is intuitively the best answer to the non-applicability of GFRestimating equations to certain populations. In the same context, and with our study finding of around half of the participants having estimated GFR less than $90 \mathrm{ml} / \mathrm{min}$ through these equations, we recommend that estimated GFR equations need to be validated in South Asian population.

\section{CONCLUSION}

This is the first published study from Pakistan that has compared all three equations available so far for estimation of GFR. The latest CKD-EPI equation was also never validated or compared with other equations in South Asia earlier. In our study, although validation of eGFR by measuring true glomerular filtration rate using inulin was not possible due to limited resources, but the statistical analysis has revealed three important results. Firstly, around half of the participants of our cohort have shown estimated GFR less than $90 \mathrm{ml} / \mathrm{min}$, so the values of estimated GFR in South Asia may be on the lower side as compared to western population. Secondly, the three equations available so far for estimation of GFR are associated linearly with each other but there are significant differences in the individual results. Thirdly, using eGFR to classify CKD should have a better consideration of physiological age-related decline in GFR. All of these findings necessitate having an adequately funded randomized study measuring true GFR of this population and at the same time validating eGFR equations to find out normal range of GFR in this region that may be different from the western population.

\section{ACKNOWLEDGMENT}

We acknowledge the other co-investigators, especially Farhana Amanullah, and team members of The Indus Hospital Community Cohort group, Ismat Lotia-Farrukh and Faisal S. Khan for data acquisition and collaborative effort in establishing a community cohort.

\section{REFERENCES}

[1] Levey, A.S., et al., National Kidney Foundation practice guidelines for chronic kidney disease: evaluation, classification, and stratification. Ann Intern Med, 2003. 139(2): p. 137-47.

[2] Levey, A.S., et al., Definition and classification of chronic kidney disease: a position statement from Kidney Disease: Improving Global Outcomes (KDIGO). Kidney Int, 2005. 67(6): p. 2089-100.

[3] Eknoyan, G. and N.W. Levin, K/DOQI clinical practice guidelines for chronic kidney disease: evaluation, classification, and stratification. Am J Kidney Dis, 2002. 39(2 Suppl 1): p. S1-266.

[4] Cockcroft, D.W. and M.H. Gault, Prediction of creatinine clearance from serum creatinine. Nephron, 1976. 16(1): p. 31-41.

[5] Levey, A.S., et al., A more accurate method to estimate glomerular filtration rate from serum creatinine: a new prediction equation. Modification of Diet in Renal Disease Study Group. Ann Intern Med, 1999. 130(6): p. 461-70.

[6] Levey, A.S., et al., A new equation to estimate glomerular filtration rate. Ann Intern Med, 2009. 150(9): p. 604-12.

[7] Lin, J., et al., A comparison of prediction equations for estimating glomerular filtration rate in adults without kidney disease. J Am Soc Nephrol, 2003. 14(10): p. 2573-2580.

[8] Stevens, L.A., et al., Evaluation of the modification of diet in renal disease study equation in a large diverse population. J Am Soc Nephrol, 2007. 18(10): p. 2749-57.

[9] Stevens, L.A., et al., Evaluation of the Chronic Kidney Disease Epidemiology Collaboration equation for estimating the glomerular filtration rate in multiple ethnicities. Kidney Int, 2011. 79(5): p. 55562. 
[10] Froissart, M., et al., Predictive performance of the modification of diet in renal disease and Cockcroft-Gault equations for estimating renal function. J Am Soc Nephrol, 2005. 16(3): p. 763-73.

[11] Poggio, E.D., et al., Performance of the modification of diet in renal disease and Cockcroft-Gault equations in the estimation of GFR in health and in chronic kidney disease. J Am Soc Nephrol, 2005. 16(2): p. $459-66$.

[12] Shastry, R., et al., Assessing Renal Function Using Cockcroft-Gault and Modification of Diet in Renal Disease Equations in Healthy South Indian Males-A pilot study. Asian Journal of Medical Sciences, 2011. 2: p. 185-189.

[13] Jafar, T., C. Schmid, and A. Levey, Serum creatinine as marker of kidney function in South Asians: a study of reduced GFR in adults in Pakistan. J Am Soc Nephrol, 2005. 16(5): p. 1413-1419.

[14] Srinivas, S., et al., Estimation of glomerular filtration rate in South Asian healthy adult kidney donors. Nephrology (Carlton), 2008. 13(5): p. 440-6.

[15] Jahan, F., et al., Assessing glomerular filtration rate in healthy adult potential kidney donors in Bangladesh: A comparison of various prediction equations with measured glomerular filtration rate by diethylentriamine pentaacetic acid renogram. Bangladesh Medical Research Council Bulletin, 2014. 39(2): p. 74-79.

[16] Khan, F.S., et al., The burden of non-communicable disease in transition communities in an Asian megacity: baseline findings from a cohort study in Karachi, Pakistan. PLoS One, 2013. 8(2): p. e56008.

[17] Alam, A., et al., Prevalence and risk factors of kidney disease in urban Karachi: baseline findings from a community cohort study. BMC Res Notes, 2014. 7(1): p. 179.

[18] Jafar, T.H., et al., Level and determinants of kidney function in a South Asian population in Pakistan. Am J Kidney Dis, 2011. 58(5): p. 76472.

[19] Raju, D., D. Lalitha, and P. Kiranmayi, Observation of estimated GFR in the assessment of chronic kidney disease: application and practice. Asian J Pharm Clin Res, 2012. 5(4): p. 201-206.

[20] Stevens, L.A., et al., Comparative Performance of the CKD Epidemiology Collaboration (CKD-EPI) and the Modification of Diet in Renal Disease (MDRD) Study Equations for Estimating GFR Levels Above $60 \mathrm{~mL} / \mathrm{min} / 1.73 \mathrm{~m}<$ sup $>2</$ sup $>$. Am J Kidney Dis, 2010. 56(3): p. 486-495.

[21] Giavarina, D., et al., Comparison of estimated glomerular filtration rate (eGFR) using the MDRD and CKD-EPI equations for CKD screening in a large population. Clin Nephrol, 2010. 74(5): p. 358-363.

[22] Matsushita, K., et al., Risk implications of the new CKD Epidemiology Collaboration (CKD-EPI) equation compared with the MDRD Study equation for estimated GFR: the Atherosclerosis Risk in Communities (ARIC) Study. Am J Kidney Dis, 2010. 55(4): p. 648-59.

[23] Rule, A.D., The CKD-EPI equation for estimating GFR from serum creatinine: real improvement or more of the same? Clin J Am Soc Nephrol, 2010. 5(6): p. 951-3.

[24] Shafi, T., et al., Comparing the association of GFR estimated by the $C K D-E P I$ and MDRD study equations and mortality: the third national health and nutrition examination survey (NHANES III). BMC Nephrol, 2012. 13(1): p. 42.

[25] Poggio, E.D., et al., Demographic and clinical characteristics associated with glomerular filtration rates in living kidney donors. Kidney Int, 2009. 75(10): p. 1079-87.

[26] Rule, A.D., et al., The association between age and nephrosclerosis on renal biopsy among healthy adults. Ann Intern Med, 2010. 152(9): p. 561-567.

[27] Glassock, R.J. and C. Winearls, Ageing and the glomerular filtration rate: truths and consequences. Trans Am Clin Climatol Assoc, 2009. 120: p. 419-28.

[28] Delanaye, P., et al., Normal reference values for glomerular filtration rate: what do we really know? Nephrol Dialys Trans, 2012. 27(7): p. 2664-2672.

[29] Kawamoto, R., et al., An association between body mass index and estimated glomerular filtration rate. Hypertension Research, 2008. 31(8): p. 1559-1564.

[30] Nagel, G., et al., Body mass index and metabolic factors predict glomerular filtration rate and albuminuria over 20 years in a high-risk population. BMC Nephrol, 2013. 14(1): p. 177.

[31] Li, Y., et al., Body mass index and annual estimated GFR decline in Chinese adults with normal renal function. Eur J Clin Nutr, 2014.

[32] Huang, W.H., et al., High body mass index reduces glomerular filtration rate decline in type II diabetes mellitus patients with stage 3 or 4 chronic kidney disease. Medicine (Baltimore), 2014. 93(7): p. e41.

[33] Delanaye, P., H. Pottel, and R. Botev, Con: Should we abandon the use of the MDRD equation in favour of the CKD-EPI equation? Nephrol Dialys Trans, 2013. 28(6): p. 1396-1403.
[34] Levey, A.S. and L.A. Stevens, Estimating GFR using the CKD epidemiology collaboration (CKD-EPI) creatinine equation: more accurate GFR estimates, lower CKD prevalence estimates, and better risk predictions. Am J Kidney Dis, 2010. 55(4): p. 622.

[35] Kidney Disease: Improving Global Outcomes., KDIGO 2012 clinical practice guideline for the evaluation and management of chronic kidney disease. 2013, New York, NY: Nature Publ. Group.

[36] Delanaye, P. and C. Mariat, The applicability of eGFR equations to different populations. Nat Rev Nephrol, 2013. 9(9): p. 513-22.

[37] Stevens, L.A., et al., Assessing kidney function--measured and estimated glomerular filtration rate. N Engl J Med, 2006. 354(23): p. 2473-83. 